\title{
Trade-off Analysis and Design of a Hydraulic Energy Scavenger
}

\author{
Enrico Zenerino, Joaquim Girardello Detoni, Diego Boero, \\ Andrea Tonoli and Marcello Chiaberge
}

Additional information is available at the end of the chapter

http://dx.doi.org/10.5772/50719

\section{Introduction}

In the last years there has been a growing interest in intelligent, autonomous devices for household applications. In the near future this technology will be part of our society; sensing and actuating will be integrated in the environment of our houses by means of energy scavengers and wireless microsystems. These systems will be capable of monitoring the environment, communicating with people and among each other, actuating and supplying themselves independently. This concept is now possible thanks to the low power consumption of electronic devices and accurate design of energy scavengers to harvest energy from the surrounding environment.

In principle, an autonomous device comprises three main subsystems: an energy scavenger, an energy storage unit and an operational stage. The energy scavenger is capable of harvesting small amounts of energy from the surroundings and converting it into electrical energy. This energy can be stored in a small unit like a small battery or capacitor, thus being available as a power supply. The operational stage can perform a variety of tasks depending on the application.

Inside its application range, this kind of systems presents several advantages with respect to devices that exploit external energy supplies. They can be simpler to employ and install, as no external connections are needed; they are environmentally friendly and might be economically advantageous in the long term. Furthermore, their autonomous nature permits the use in locations where the local energy grid is not present and allows them to be 'hidden' in the environment, being independent from interaction with humans.

The idea is to make autonomous and more energy efficient processes in some very specific areas, particularly in the management of household heating/cooling systems, and in the 
environmental monitoring. The basic concept is to convert a fraction of the energy that would be normally dissipated by the process into electrical energy. These "secondary" energy sources are then used as primary sources in micro generators whose electrical current will be used to power the devices distributed along the process. The physical phenomena involved in this energy conversion can be essentially: the piezoelectric, photovoltaic and thermoelectric effects and phenomena related to fluid dynamics. Significant examples of energy harvesting are evident in particular in the following scientific fields: the construction of electric generators coupled to microturbines (Chunyan et al., 2010), (Bansal et al, 2009), (Yan et al., 2011), (Zainuddin, H et al., 2009); the Stirling thermodynamic cycle (Valdes, 2004), the Seebeck effect in thermoelectric generators (Lineykin et al., 2007), (Lu et al., 2010) and an Helmholtz resonator based generator (Kim et al. 2009).

Within this context the objective of this work is to present a trade-off analysis between different types of hydraulic machines, electric generators and energy storage units to reach a good compromise in the design of harvesting devices to be integrated in fluid distribution systems.

\section{System description}

The present section describes the system configuration of the energy harvester (or scavenger) used to supply a thermostatic motorized valve of a heating system for residential applications.

The Scavenger of Fig. 1 is composed by three main subsystems:

- hydraulic machine

- electric generator

- $\quad$ energy storage unit

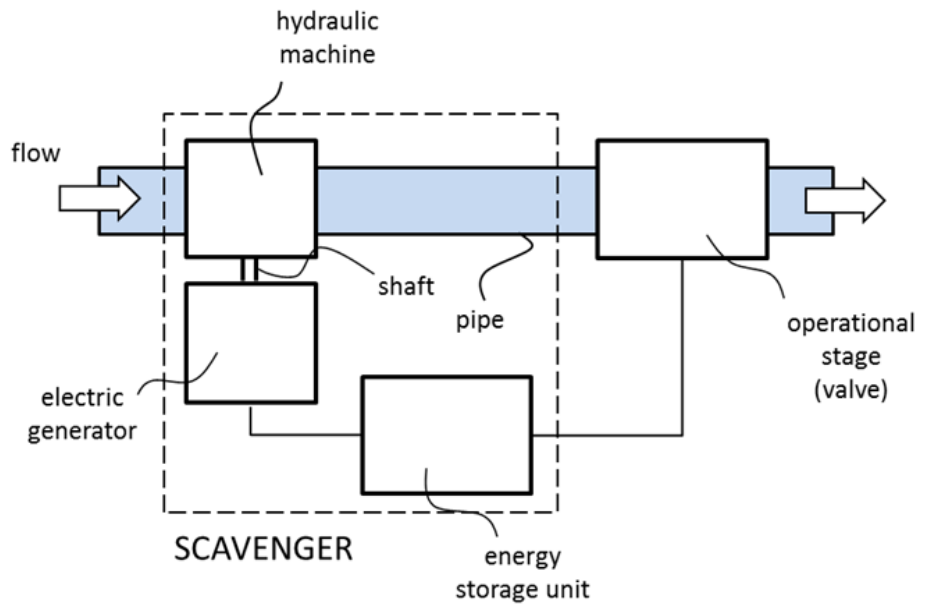

Figure 1. Hydraulic Energy Harvester system scheme 
A cross flow turbine transforms the hydraulic power of the water flow into mechanical power used to drive a small electric generator. The device includes an energy storage unit to match the relatively constant power production profile of the generator unit with the more discontinuous one that characterizes the load. Additionally, since the generator and the valve are in series, the energy storage ensures the possibility to open the valve from the closed state (no flow). The energy is then stored during the peaks of production and then reuse it in a second time when prompted by the operational unit, the valve in our case. To this end the power management system is made to operate at its Maximum Power Point (MPPT) through closed loop control of recovered current from the scavenger unit.

\subsection{Specifications and design choices}

The collection of specifications starts from the hydraulic data available for the system from which we want to extract the energy. The typical flow rate of household heating system pipelines is between 1.3 and $4 \mathrm{l} / \mathrm{min}$. The geometrical size of the device must be compliant with the available space at the interface between the heating element of conditioning systems and the pipeline. Other specifications are related to electrical power and voltage that must be generated. A campaign of experimental tests performed on motorized thermostatic valves shows that the average power is about $100 \mathrm{~mW}$ with peaks that can reach $1 \mathrm{~W}$ for a short time during valve actuation. The minimum nominal voltage must be compatible to the voltage generated by a couple of AA-type batteries currently used to power the electronic thermostatic valves actuation units. Table 1 lists the above-mentioned specifications.

\begin{tabular}{lccc}
\hline \multicolumn{1}{c}{ Parameter } & Symbol & Value & Unit \\
\hline water nominal flow rate & $Q$ & 2 & $1 / \mathrm{min}$ \\
radial size & $r$ & $<60$ & $\mathrm{~mm}$ \\
axial size & $l$ & $<90$ & $\mathrm{~mm}$ \\
min nominal voltage generated & $V_{n}$ & $>3$ & $\mathrm{~V}$ \\
nominal power generated & $P_{n}$ & 100 & $\mathrm{~mW}$ \\
\hline
\end{tabular}

Table 1. System specification

In addition to specification some key choices are taken to proceed with the design, they are summarised in Tab. 2. One aspect which strongly influences the design of the system is the size of the inlet nozzle of the hydraulic machine. This choice is driven by the need to avoid chocking of the nozzle because of the large amount of dirt particles that characterize the fluid of heating systems. The design choice is to have a nozzle diameter $d$ larger than $4 \mathrm{~mm}$, as shown in the cross-section view of Fig. 17. Moreover a relatively large inlet nozzle diameter reduces the hydraulic losses due to the introduction of the scavenger into the heating system. The advantage is of reducing the need of increasing the size of the main pump that produces the hydraulic flow in the system. Another design choice about the realization of the turbine concerns the speed rotation of the runner. Here, a rated nominal speed of $1000 \mathrm{rpm}$ has been chosen for the device. This rotation speed should be compatible 
with the precision that can be reached with standard production process of some details of the generator unit as impeller and bushings. A limited angular speed also ensures an adequate degree of durability and strength. This speed value is precautionary as regards the possibility of creating vibrations, that may occur at higher speeds and lead to the failure of the rotating parts.

\begin{tabular}{lccc}
\hline Parameter & Symbol & Value & Unit \\
\hline inlet nozzle diameter & $d$ & $>4$ & $\mathrm{~mm}$ \\
generator rated speed & $n$ & 1000 & $\mathrm{rpm}$ \\
\hline
\end{tabular}

Table 2. Design choices

\section{Trade off and design}

This phase is carried on by splitting the system in its three subsystems. Following the transformations of energy that take place in the device the hydraulic machine is met first, then the electric machine and finally the energy storage unit, without neglecting their mutual interactions.

\subsection{Hydraulic machine}

We start from the conversion of the kinetic energy of the water into mechanical rotational energy of the hydraulic machine runner. Different typologies of machine are considered for the hydraulic machine: water turbines for mini and micro power plant and a gear pump. The water turbines for micro-hydropower generation can be classified into two main categories: impulse turbines and reaction turbines as described in (Inversin 1994) and listed in Fig. 2. In impulse turbines there is no expansion of the flow within the moving blades of the runner and, as such, the pressure remains constant while passing over the blades. In reaction turbines the stream expands as it flows over the blades, therefore producing a drop in pressure which gives a reaction and hence motion to the rotor.

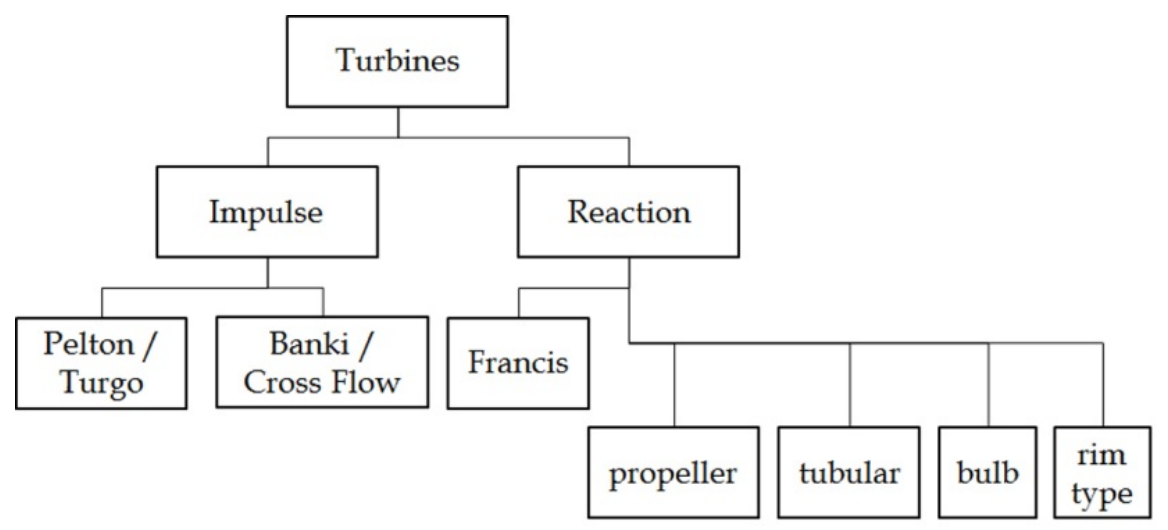

Figure 2. Classification of turbines for micro-hydropower generation 
In general, impulse turbines are used for high head plants while reaction turbines for low head sites.

The turbines available on the market are usually very large compared to what needed for the present application, so a new turbine needs to be designed following the indications and design criteria of classical hydropower turbines of much larger dimensions.

For a given nominal output rated power and flow rate, equation (1) allows evaluating the pressure drop across the hydraulic machine:

$$
P_{n}=Q \cdot \Delta p
$$

Then, from the energy conservation principle of an ideal fluid inside a pipeline (i.e. the Bernoulli's principle) it is possible to determine the head of the water flow $H$.

$$
\Delta p=\rho \cdot g \cdot H
$$

From the head $H$, the absolute mean speed c of the water jet at the nozzle output can be found following the classical design indications of a Pelton turbine:

$$
c=\varphi \cdot \sqrt{2 \cdot g \cdot H}
$$

where $\varphi$ is the nozzle flow factor, evaluated in the present case as equal to 0.97 . As reported in (Nechleba, 1957), the best efficiency is reached when the circumferential speed of the runner (also indicated as drag speed) is related to the water jet speed as follows:

$$
u=0.46 \cdot c
$$

The circumferential speed $u$ can then be related to the diameter $A$ of the rotor and to the angular speed $n$ of the runner:

$$
A=\frac{60 \cdot u}{\pi \cdot n}
$$

where $u$ is expressed in meter per second and $n$ in revolutions per minute.

A trade-off analysis has been performed between different kind of turbines by making the preliminary design of the runner. The case of hydraulic gear motor has been included in the trade off study along with the classical Francis, Pelton, and Banki turbines. The results of the preliminary design are presented in Tab. 3. All of them are compatible in size with the design specifications.

In addition to the preliminary design, other critical aspects related both to functionality and practical feasibility have been considered in the trade-off. In particular, the overall dimensions of the different layouts have been evaluated along with the possibility to integrate the rotor of the electric machine to that of the turbine, and with the need of avoiding chocking due to the debris transported by the water flow. Finally, the constructive complexity of the different parts has also been considered, in particular focusing on rotor 
and stator. Each of these aspects have been ranked in a three step scale. The results of this trade-off are reported in Tab. 4.

\begin{tabular}{lcccc}
\hline Hydraulic machines & \multicolumn{3}{c}{ Turbines } \\
& Francis & Pelton & Banki & Gear Pump \\
\hline pressure drop $(\Delta p)$ & & \multicolumn{2}{c}{$4200 \mathrm{~Pa}$} & \\
head $(H)$ & & $0.43 \mathrm{~m}$ & \\
nozzle jet speed $(c)$ & $2.17 \mathrm{~m} / \mathrm{s}$ & $2.78 \mathrm{~m} / \mathrm{s}$ & $2.84 \mathrm{~m} / \mathrm{s}$ & \\
circ. runner speed $(u)$ & $1,65 \mathrm{~m} / \mathrm{s}$ & $1.33 \mathrm{~m} / \mathrm{s}$ & $1.33 \mathrm{~m} / \mathrm{s}$ & \\
Runner diameter $(A)$ & $31 \mathrm{~mm}$ & $25 \mathrm{~mm}$ & $24 \mathrm{~mm}$ & \\
Displacement $(V)$ & & & & $2,5 \mathrm{~cm}^{3} / \mathrm{turn}$ \\
\hline
\end{tabular}

Table 3. Preliminary design of hydraulic machines. Comparison of the main parameters of the different solutions.

\begin{tabular}{lcccc}
\hline Hydraulic machines & \multicolumn{3}{c}{ Turbines } & Volumetric \\
\cline { 2 - 4 } runner & Reaction & \multicolumn{2}{c}{ Action } & \\
\cline { 2 - 5 } & Axial & \multicolumn{2}{c}{ Tangential } & \\
\hline & Francis & Pelton & Banki & Gear Pump \\
\hline Radial size & $* *$ & $* *$ & $* *$ & $*$ \\
Axial size & $* *$ & $* *$ & $* * *$ & $*$ \\
Rotor complexity & $*$ & $*$ & $* * *$ & $* * *$ \\
Stator complexity & $*$ & $* *$ & $* * *$ & $*$ \\
Electric machine interface & $*$ & $* *$ & $* * *$ & $*$ \\
Choking risk & $*$ & $* *$ & $* *$ & $*$ \\
\hline
\end{tabular}

*** good, ${ }^{* *}$ average, ${ }^{*}$ bad

Table 4. Trade-off analysis between hydraulic machines.

\subsubsection{Cross Flow or Banki turbine design}

The Cross Flow or Banki turbine appears to be the best in almost all aspects examined. Its strengths are the simplicity of construction, the compact size, a good interfacing capability with the generator and a limited risk of choking.

Following the Banki water turbine theory reported in (Mockmore et al. 1959) the two main parts of the turbine, namely the nozzle and the runner, have been designed. The design drawings of the Banki turbine runner are shown in Fig. 3, and its characteristic parameters are given in Tab. 5 . 
a)

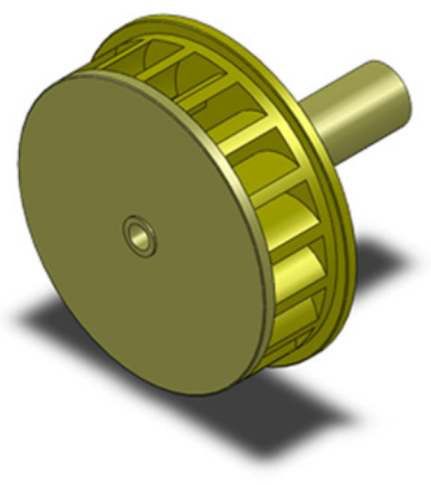

b)

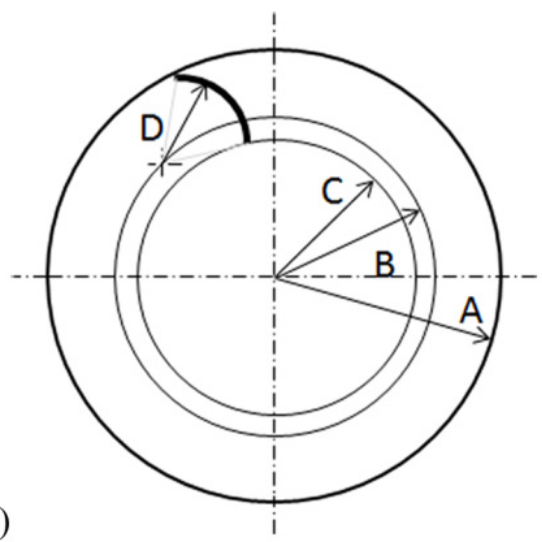

Figure 3. Banki turbine runner: a) 3D model. b) geometrical parameters.

\begin{tabular}{lccc}
\hline parameter & symbol & value & unit \\
\hline inlet nozzle diameter & $d$ & 4 & $\mathrm{~mm}$ \\
runner diameter & $A$ & 24 & $\mathrm{~mm}$ \\
center of blade curvature diameter & $B$ & 17.7 & $\mathrm{~mm}$ \\
blade root diameter & $C$ & 15.8 & $\mathrm{~mm}$ \\
blade curvature radius & $D$ & 7.8 & $\mathrm{~mm}$ \\
runner breadth & $L$ & 5 & $\mathrm{~mm}$ \\
number of blades & $N$ & 18 & - \\
\hline
\end{tabular}

Table 5. Cross flow or Banki turbine construction parameters.

\subsection{Electric generator}

In order to convert the rotational mechanical energy from the turbine shaft into electrical energy which can be used to power the wireless spot and operate the valves of the system, a miniaturized electrical generator has been designed especially for this application. Two different configurations of generators have been investigated in order to obtain a clear perspective on the advantages and drawbacks of each one. The first configuration is based on a multiphase permanent magnet generator layout. The second is a single phase permanent magnet generator having claw pole structure. Fig. 4 shows the two different configurations describing the main components of the electrical machines, namely, rotating permanent magnet (1), generator's coil (2), and stator yoke (3).

Both cases consider permanent magnet excitation on the rotor. It is known that for reduced size applications such as the present one, it is better to use permanent magnet excitation instead of electrically excited magnetic systems. The electrical excitation is disadvantageous in these cases owing to unfavourable scaling of the currents (Arnold 2007). 
a)

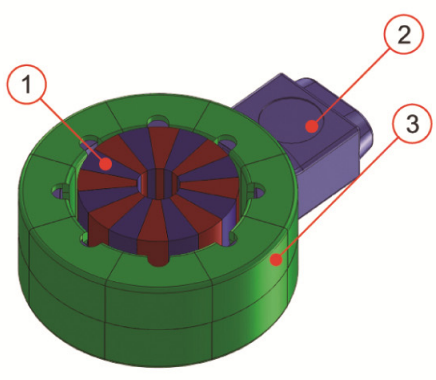

b)

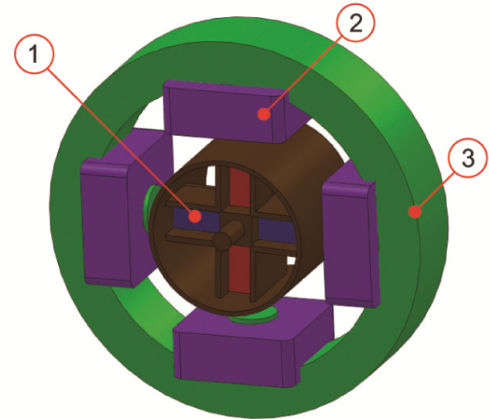

Figure 4. Configurations of electrical machines studied during the trade-off analysis. a) Two phase generator; b) Claw pole generator.

A trade-off analysis is performed using virtual prototyping tools. The difficulties related to the mechanical layouts were studied using CAD models while the electrical and magnetic properties were analyzed using analytical and finite element (FE) models.

\begin{tabular}{|c|c|c|}
\hline & $\begin{array}{l}\text { Configuration } 1 \\
\text { Multiphase }\end{array}$ & $\begin{array}{l}\text { Configuration } 2 \\
\text { Claw pole }\end{array}$ \\
\hline Rotor complexity & $* * *$ & $* * *$ \\
\hline Stator complexity & * & $* *$ \\
\hline Overall volume & * & $* *$ \\
\hline Number of pole pairs & * & $* * *$ \\
\hline Winding complexity & $* *$ & $* * *$ \\
\hline Detent torque & * & $* *$ \\
\hline
\end{tabular}

Table 6. Comparison between multiphase and claw pole layouts.

From these models it is possible to obtain a relatively accurate perspective of the critical aspects related to the feasibility of each configuration of the generator. From the application point of view, the most important characteristics are compared in Tab. 6. Analyzing the table, it is easy to conclude that the claw pole configuration is more suitable for this application. Its layout makes it possible to obtain a simple and compact structure, and, since the output voltage must be rectified to supply the batteries, there is no advantage in having a multiphase winding, such as configuration 1. Furthermore, the single phase winding enables having a larger number of magnetic pole pairs, thus resulting in an increase of the frequency of the induced electromotive force (EMF), which is beneficial from the electronic point of view. Another aspect that cannot be neglected is the amplitude of the detent torque generated by the interaction between rotor's permanent magnets and stator's yoke (Lossec et al. 2010). A larger number of pole pairs tends to reduce the amplitude of the detent torque for the same rotor radius since the slot opening is reduced (Hendershot et al 1994). Moreover, the geometry of the teeth in the claw pole configuration can be adjusted in order 
to further reduce the cogging torque. On the other hand, the multiphase configuration creates problems in this aspect due to the difficulty in realizing yoke and windings having such small dimensions.

\subsubsection{Finite element modeling}

The prediction of the generator's performance is developed by means of FE simulations. The simulations are performed using a stationary formulation without electric currents for one single pole pair of the electrical machine. Non-linear magnetic properties were considered in the iron parts of the structure. Fig. 5 shows the model used in the finite element modeling, evidencing the use of cyclic symmetry boundary conditions to improve the modeling quality with reduced computational cost. The analyses are conducted in order to calculate the flux linking the coil for different values of rotor angles. This information is then used to evaluate the induced voltage with respect to the rotor's spin speed. To this end, the problem is set to allow the rotor mesh to move with respect to the stator mesh, thus enabling the calculation of the magnetic quantities for different values of angular position between the two. Notice that the air surrounding the rotor and stator of the electrical machine is modeled, but is not shown in the illustration. Fig. 6 shows the results obtained from the FE model evidencing the path of flux lines inside the stator's yoke (Fig. 6b). Moreover, it can be noticed that the flux densities inside the iron are relatively low (Fig. 6a) resulting in very little or no saturation.

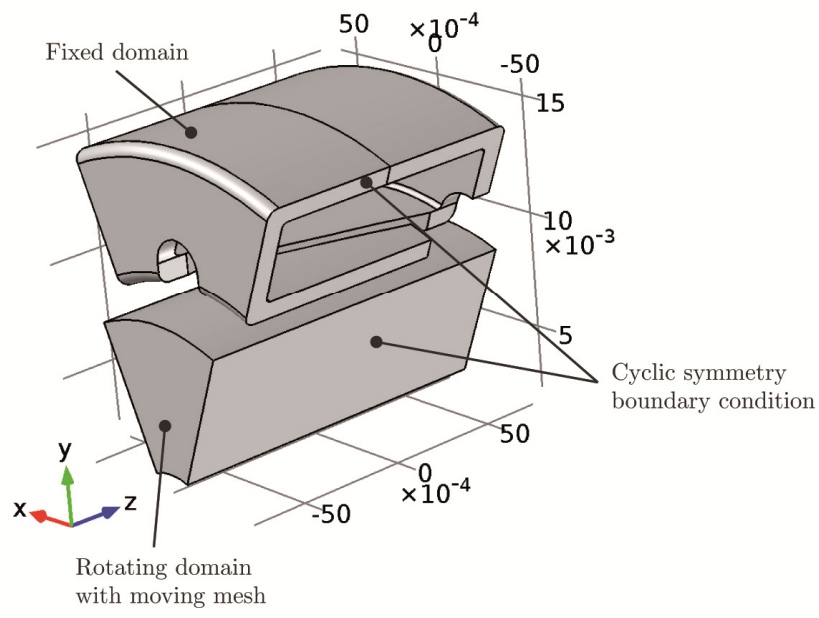

Figure 5. Settings of the FE model for the magnetic simulations.

The parameters characterizing the system described in the FE simulations are summarized in Tab. 7, and the flux linkage wave calculated with the FE model is illustrated in Fig. 7. Observing the graph it can be noticed that the flux linking the coil realizes one complete period every 45 mechanical degrees, evidencing the existence of eight magnetic pole pairs. 
Furthermore, the flux linkage is a sinusoidal function of the rotor angle and, since the generator operates at constant rotating speed during most of its operative life, the analysis can be performed in terms of RMS quantities.

The velocity constant $k_{e}$ can be obtained from the data plotted in Fig. 7 considering the flux $\lambda$ linkage derivative with respect to the rotor angle $\theta$ as:

$$
k_{e}=\frac{1}{\sqrt{2}} \frac{\partial \lambda}{\partial \theta}
$$

The induced electromotive force $e$ can then be obtained as:

$$
e=k_{e} \omega
$$

where $\omega$ is the generator rotational speed expressed in radians per second.The value of the velocity constant obtained for the generator is reported in Tab. 7.

\begin{tabular}{lccc}
\hline Parameter & Symbol & Value & Unit \\
\hline Stator's outer diameter & $D_{e}$ & 31.5 & $\mathrm{~mm}$ \\
Stator's inner diameter & $D_{i}$ & 20 & $\mathrm{~mm}$ \\
Rotor's outer diameter & $d_{e}$ & 19 & $\mathrm{~mm}$ \\
Active length & $l_{a}$ & 12.5 & $\mathrm{~mm}$ \\
Air gap length & $t$ & 0.5 & $\mathrm{~mm}$ \\
Number of pole pairs & $p$ & 8 & - \\
Coil turns & $N$ & 1200 & - \\
Permanent magnet induction & $B_{r}$ & 0.42 & $\mathrm{~T}$ \\
Velocity constant & $k_{e}$ & 9.41 & $\mathrm{~V} / \mathrm{krpm}$ \\
\hline
\end{tabular}

Table 7. Claw pole generator nominal parameters.

Magnetic flux density norm (T)

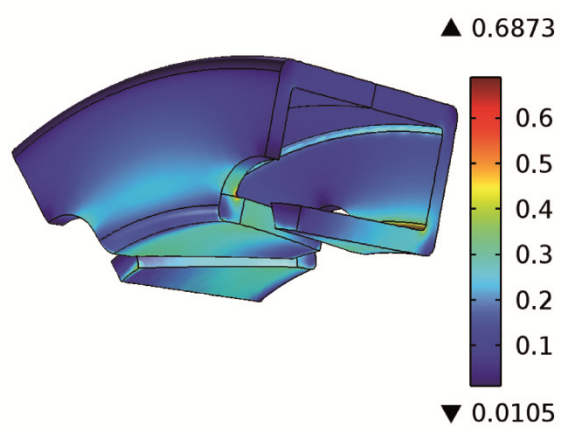

Magnetic flux density (Spatial)

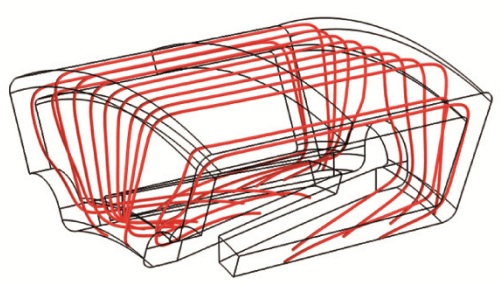

b)

Figure 6. Results of the finite element simulations on the claw pole generator. a) Magnetic flux density in the stator in teslas $(\mathrm{T})$; $\mathrm{b}$ ) magnetic flux lines inside the iron. 


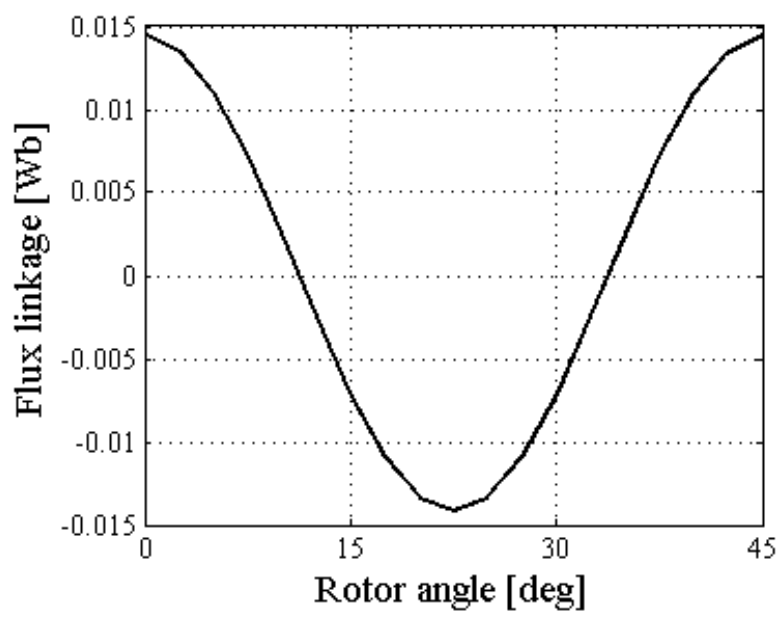

Figure 7. Flux linking the generator's windings for different values of rotor angle.

\subsection{Energy storage unit}

In order to properly manage the energy coming out from the generator, it is necessary to consider that the maximum amount of power is generated when the impedance of the load is nearly equal to the impedance of the generator. Due to this limitation it is important to design a control system shown in Fig. 8 that can monitor, manage and store the energy in order to increase the efficiency of the whole system. For this reason the energy converter can be divided into several subsystem:

- rectifier

- $\mathrm{DC} / \mathrm{DC}$ regulator

- $\quad$ storage system

- control system

The main idea was to develop each single subsystem in order to have more degree of freedom for each subsystem: active rectifiers to reduce energy losses and perform a Power Factor Corrector (PFC) regulation, independent DC/DC regulator with different Maximum Power Point Tracker (MPPT) according to the instantaneous situation, charge controller to ensure a good storage reduce as much as possible the memory effects in the battery, and a unique controller to manage properly all the interaction and the functions of these systems.

Due to the limited time for testing and to simplify the construction of the first prototype, an integrated solution shown in Fig. 9 is preferable to reduce cost and to obtain a suitable industrial solution. For these reason some solution from Linear Technologies (LT) turned out to be useful because they include into a single chip the DC/DC regulator, the charge controller and the main control system, therefore reducing cost and implementation time. Only few additional components have been selected and added to the integrated chip to obtain the final requested solution. 


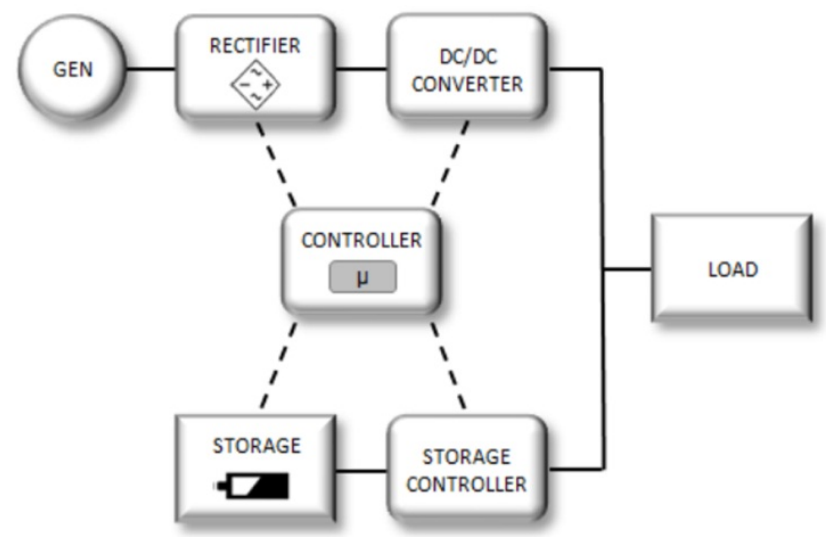

Figure 8. Architecture component interaction and interconnection.

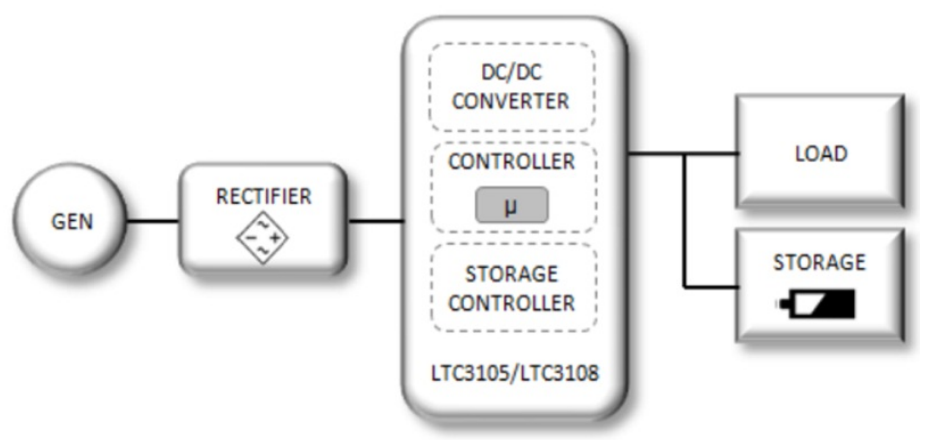

Figure 9. Block diagram of the integrated solution with LT components

The electric generator produces a sine wave with an electric frequency proportional to the mechanical velocity of the hydraulic turbine $n$ expressed in rpm with the following relation:

$$
f_{e}=\frac{n}{60} p
$$

where $p$ is the number of the pole pairs as reported in Tab. 7 .

The easiest way to convert this sinusoidal voltage into continuous voltage is using a rectifier bridge; the ideal solution is based on active rectifier to obtain a voltage drop as lower as possible, but for this application, during the first tests, the results were not so different using a traditional passive rectifier instead. For this reason the first prototype was developed using a simple single phase rectifier composed by four Schottky diodes to reduce as much as possible the power losses. Using BAT54 diodes the voltage drop is about $250 \div 400 \mathrm{mV}$, equal to $500 \div 800 \mathrm{mV}$ for each stage of conversion according to the current flow. A 10uF capacitor is enough to keep constant the voltage with a low output ripple $(<1 \%$ of the maximum peak voltage). 
The second stage is the regulator used to increase or reduce the input voltage to provide an output voltage around $3 \div 3.3 \mathrm{~V}$ to properly supply any kind of microprocessor or actuator. Using a buck-boost converter it is easy to satisfy this requirement but, due to the lower amount of energy generated by the turbine, it is necessary to implement also the MPPT algorithm. The Maximum Power Point Tracker is an algorithm normally used in photovoltaic cells; its role is to constantly check the input voltage and current to know exactly how much power is available and to limit the current absorption from the generator so as to keep always the condition of maximum production (see Fig. 10). To perform this operation it is necessary to use two different feedbacks: the first is used to check the output voltage to keep it constant with a low ripple; the second one checks the input current, limiting its absorption by varying the duty cycle of the DC/DC converter. In this way even if a very heavy load, alike a completely discharged battery, is connected, the converter can make the generator work in the most efficient condition. This algorithm needs to be included into the general control system alike an independent microprocessor or an integrated chip. The use of a microprocessor allows to modify these conversion algorithms without hardware changes, but only varying the internal control parameters.

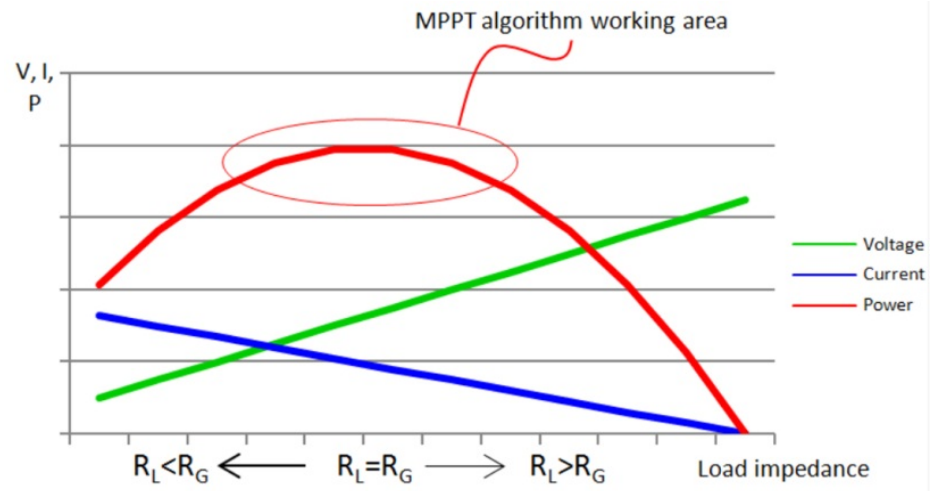

Figure 10. Current adjustment from MPPT to achieve maximum efficiency

In this system, the recovered energy can be used directly by the loads or can be partially stored for future use. The storage system can be divided in two main components based on different technologies:

- electrostatic storage ( super capacitors): most suitable when the load requires small quantity of energy in short interval (less than $5 \div 15$ minutes) and the generator can provide always the average energy required. The auto-discharge factor is higher than in the chemical solution but in the short period is a value than can be neglected.

- chemical storage ( NiMH o Lilon batteries): most suitable if the generator has an intermittent production or the load requires medium/high quantity of energy with long pause interval also when the generator is switched off. The auto-discharge factor is low but the storage efficiency is lower than in the electrostatic storage, causing efficiency reduction during charge and discharge. 
If the load is composed by a combination of continuous small absorptions with occasional high requests of energy, it can be useful to combine the above storage technologies in order to reduce energy losses.

The first prototype was not developed using an independent control system and energy converter, but using an integrated solution were all the three elements are included into one single chip. Two different boards were developed using two chips from Linear Technologies. The first solution used the LTC3108, a buck-boost converter without MPPT algorithm but capable of converting input voltages lower than $200 \mathrm{mV}$. This solution was adopted due to the extremely low power coming out from the generator in the first prototype developed. Increasing the generator production, it was possible to move to the LTC3105 regulator, that is capable of converting input voltages higher than $500 \mathrm{mV}$ till to $5 \mathrm{~V}$, including an internal MPPT control in order to adapt the load absorption according to the generator production.

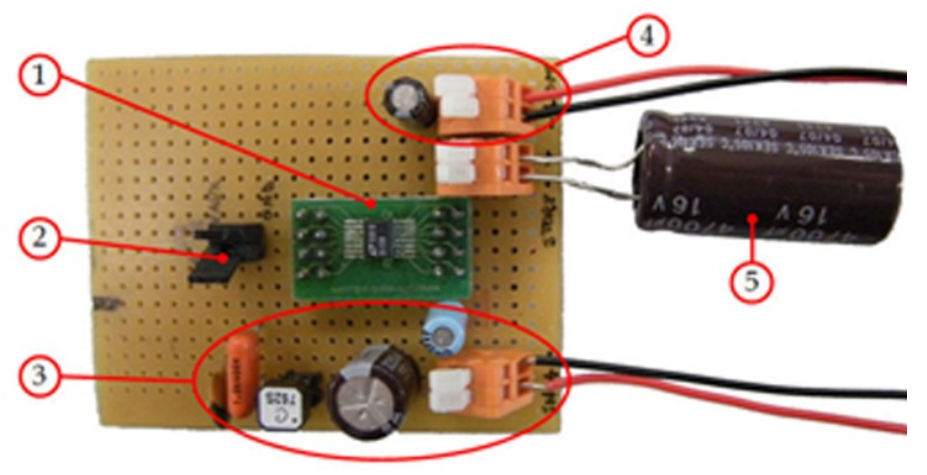

Figure 11. DC/DC converter: LTC3108 controller (1), output voltage selectors (2), input terminal, switching inductance and filters (3), regulated output $2.3 \mathrm{~V} \div 5 \mathrm{~V}(4), 5.25 \mathrm{~V}$ storage battery or capacitor (5)

In the test board two different topologies were tested. The first one used an output voltage set to $2.2 \div 2.3 \mathrm{~V}$ connected to a $1 \mathrm{~F} 2.3 \mathrm{~V}$ super-capacitor (see Fig. 11). This solution was adopted to supply a very light load like a microcontroller (PIC16F886) that run a simple code to only switch on and off one led. The second solution used an output voltage of $4 \mathrm{~V}$ to supply directly the load and to recharge the $3.6 \mathrm{~V} 220 \mathrm{~mA}$ battery; specifically, when the converter was on, the remaining current that was not used by the load was employed to recharge the battery, whereas when the input generator was off, the OUT pin was disconnected from the converter so that all the energy required was provided by the battery. A custom external controller is necessary to ensure battery protection from deep discharges $(<3 \mathrm{~V})$ during switch-off period to prevent irreversible damage to the cells.

\section{Experimental validation}

The system described in the previous sections was implemented and experimental tests were carried out to verify the correspondence between the design model and the real 
system. The experimental tests were first addressed to investigate the performance of each subsystem and in the second instance of the whole system. For this purpose several test rigs have been built in order to characterize the single components and the interactions between them. Experimental tests verified the nominal design expectations and have been used also to perform sensitivity analysis on different parameters of some components.

\subsection{Dry test rig}

To characterize the electric generator, a "dry" test rig was developed as shown in Fig. 12. It is constituted of a DC electric motor (2), which is used to drive the generator's rotor, and the generator's stator (1). A DC power supply is used to energize the drive motor and to put the generator rotor into rotation at different rotational speeds. The values of induced EMF measured during the tests are compared to the FE model in Fig. 13.

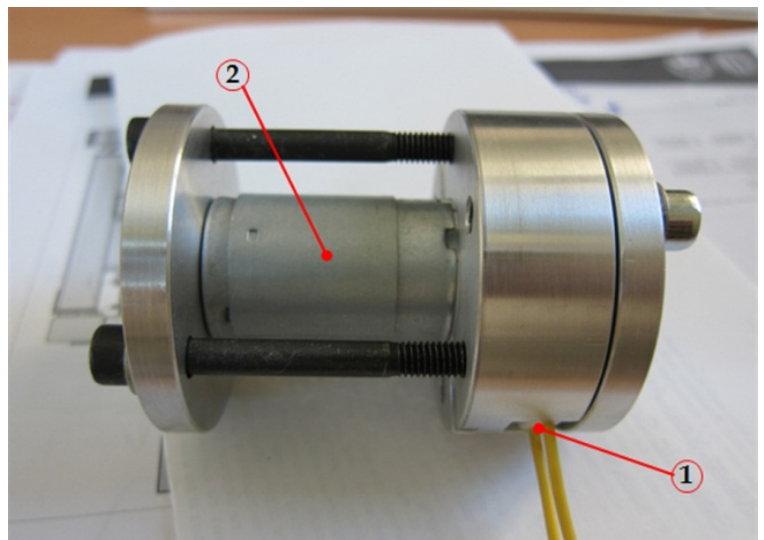

Figure 12. Dry Test Rig realization

Different configurations of the generator have been implemented and investigated with several values of the air gap $t: 0.5 \mathrm{~mm}$ for the nominal configuration as reported in Tab. 7, $1 \mathrm{~mm}$ for the preliminary supply of claw poles generators tested in the dry test rig, and $1.25 \mathrm{~mm}$ for claw poles generators compliant with the wet test rig. The experimental values represent the RMS value of the voltage measured between the two ends of the stator coil, whereas the FE model results were obtained by using Eq. 6 and Eq. 7 with the data corresponding to the above mentioned configuration. The obtained experimental results show good correlations with the numerical values resulting from the model simulation.

In Fig. 14 the generated power is plotted as function of the rotating speed of the generator for the different air gaps. It is clear that the rated generated power is significantly less than the $100 \mathrm{~mW}$ required. This is due to the fact that it was not possible to find a ferrite with a value of the permanent magnet induction equal to $0.42 \mathrm{~T}$ but only $0.27 \mathrm{~T}$, with a consequent loss in performance. It must also be emphasized the drastic lack of performance in terms of 
power generated by the increasing of the air gap from the nominal value of $0.5 \mathrm{~mm}$ up to the value of the wet test rig equal to $1.25 \mathrm{~mm}$.

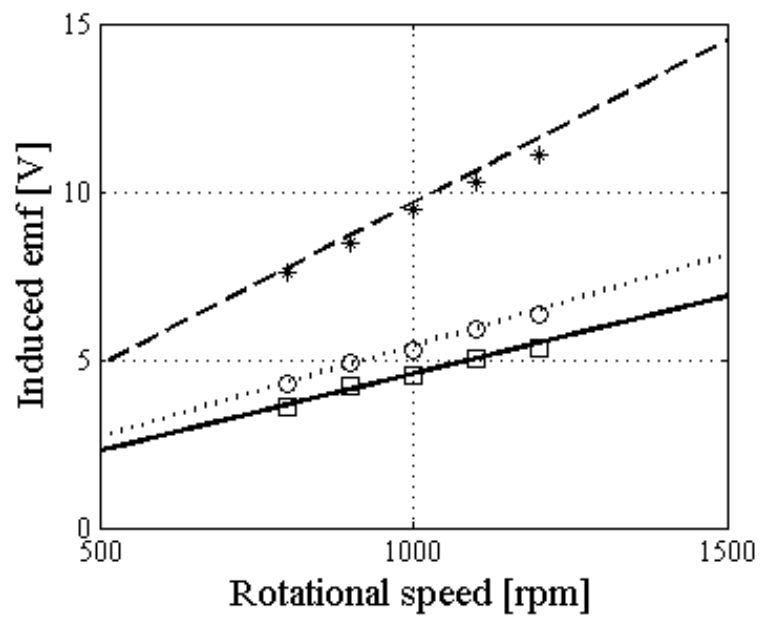

Figure 13. Induced emf for different values of the air gap : $t=0.5 \mathrm{~mm}$ (dashed line) vs exp. (star markers); $t=1 \mathrm{~mm}$ (dotted line) vs exp. (circular markers); $t=1.25 \mathrm{~mm}$ (solid line) vs exp. (square markers).

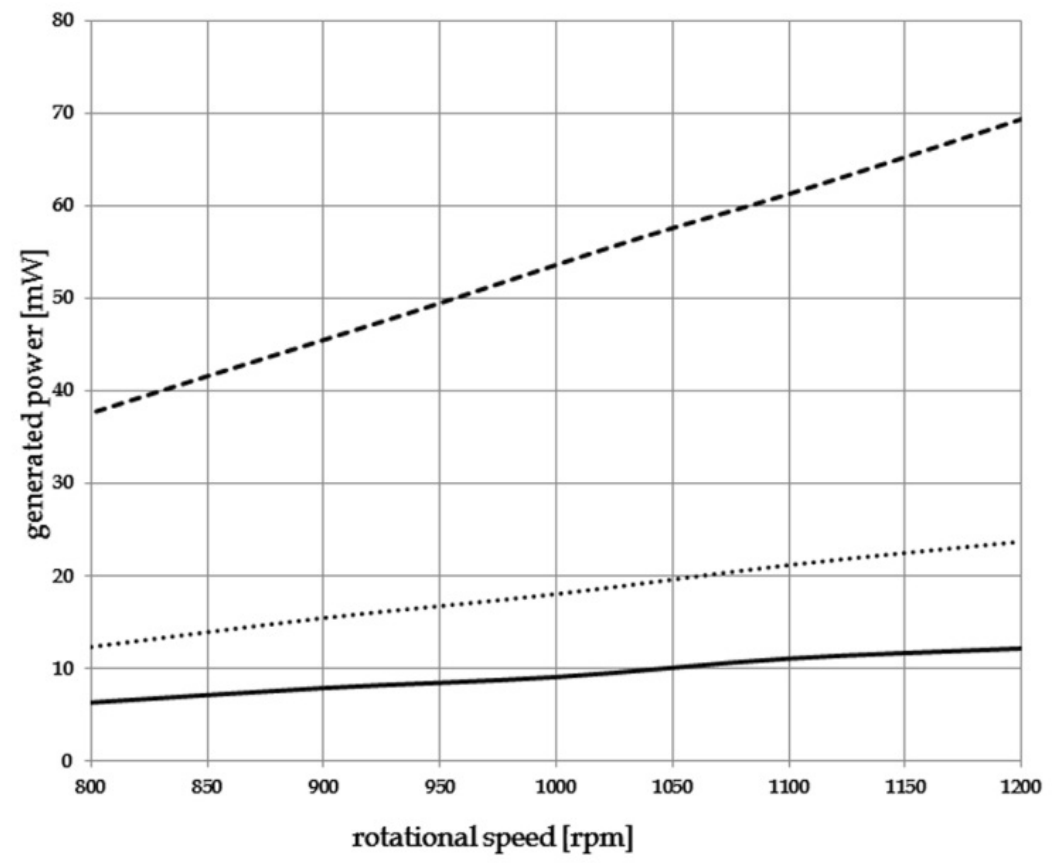

Figure 14. Air gap Sensitivity Analysis: generated power with external load equal to generator resistance. $t=0.5 \mathrm{~mm}$ (dashed line), $t=1 \mathrm{~mm}$ (dotted line), $t=1.25 \mathrm{~mm}$ (solid line). 


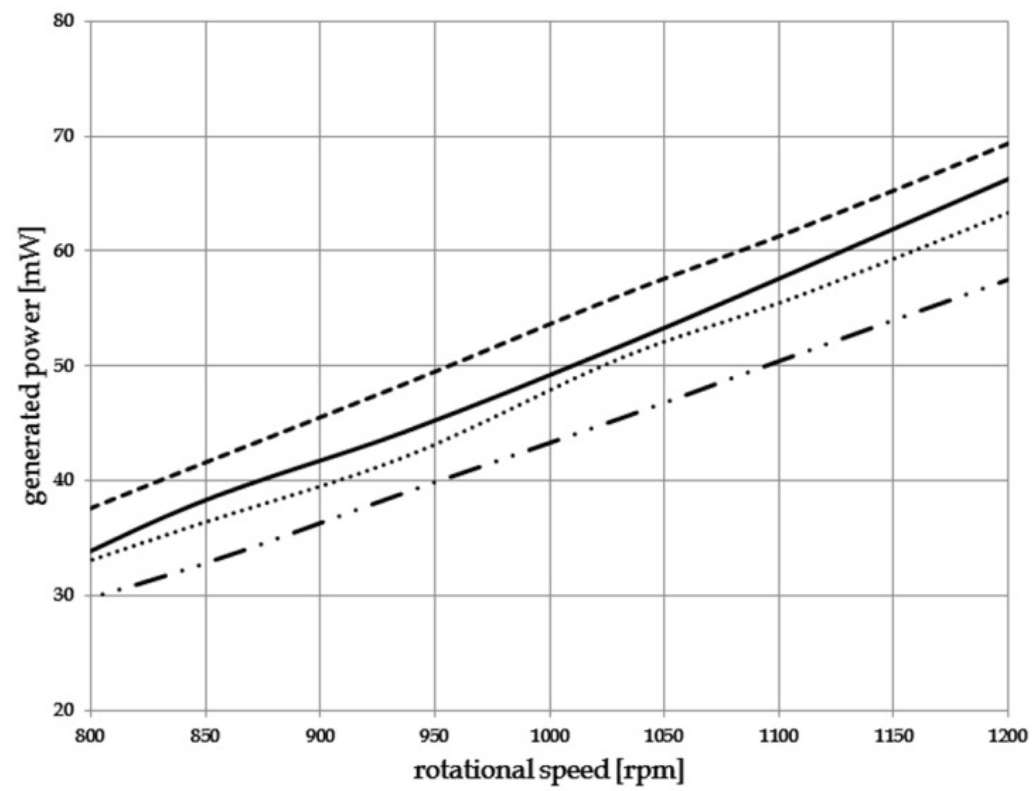

Figure 15. Number of coils Sensitivity Analysis: generated power with external load equal to generator resistance. Nominal $N=1200$ (dashed line), $N=800$ (dash dotted line), $N=620$ (dotted line) and $N=490$ (solid line).

A lower influence has been found with the variation of the number of coils of the generator stator $N$ as shown in Fig. 15, leaving a certain degree of freedom from this point of view.

\subsection{Wet test rig}

A second test rig has been developed in order to test the electrical generator coupled with the hydraulic turbine, which makes it possible to characterize both the generator and the hydraulic machine as shown in Fig. 16. This wet test rig is composed by the scavenger (1), connected in series to a flow meter (2), which are both supplied by a domestic water pipeline, where the water flow is adjustable by a tap (3). The measuring system consists of a flow meter (2), a multimeter (4) and an oscilloscope (5) in order to correlate the generated power with the available flow rate. In the lower left box of Fig. 16 an enlarged view of the rapid prototyping realization of the scavenger (1) is also shown.

In Fig. 17 a cross-section of the scavenger is reported; the device incorporates a eighteen blades Banki turbine (1) (see Tab. 5 for other specifications) and a claw-poles voltage generator (2) (see Tab. 7 for nominal specifications). The design of the integration between the two parts required special attention, in particular to ensure the sealing between rotating and fixed parts and to prevent the direct contact between the main water flow and the electrical generator. The presence of the magnet in the generator rotor and ferromagnetic 
residues in the water may lead to choking risk. To solve the problem of sealing an O-ring (4) has been introduced; to limit as much as possible the choking risk labyrinth seals have been used among the rotor housing and the runner.

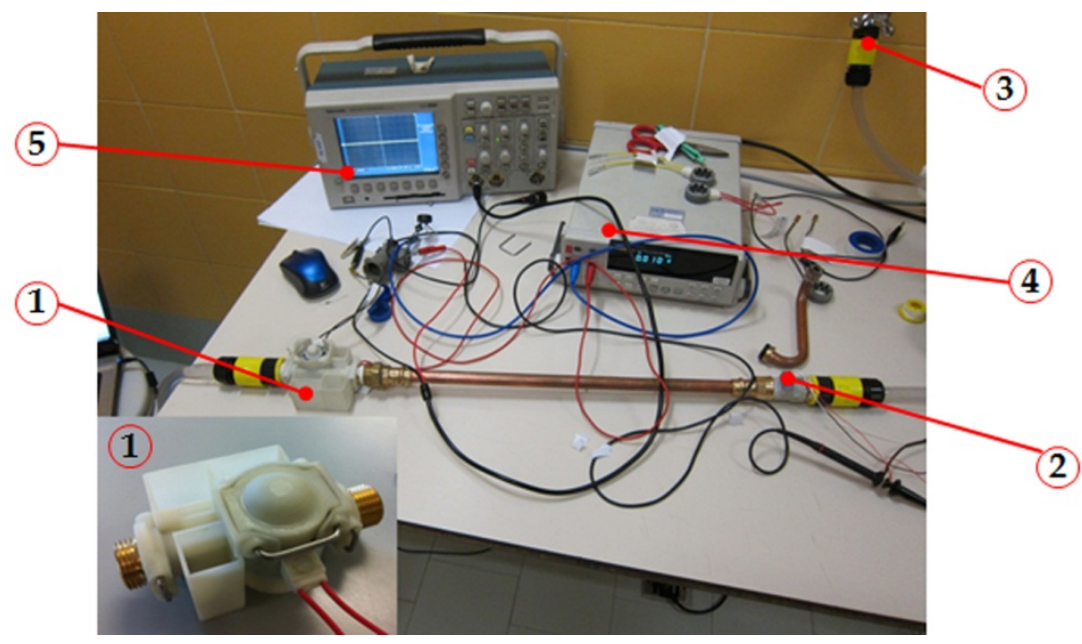

Figure 16. Wet Test Rig: scavenger (1), flow meter (2), domestic water supply and tap (3), multimeter (4) and oscilloscope (5).

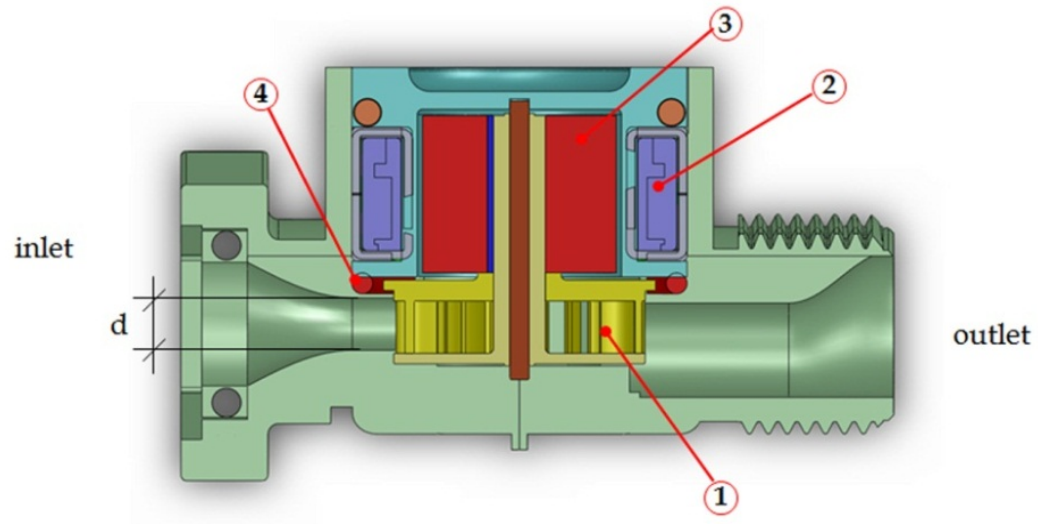

Figure 17. Cross-section view of the scavenger: Banki turbine (1), claw pole generator (2), permanent magnet rotor (3) and O-ring (4).

Owing to manufacturing problems related to the choice of the rapid prototyping process, the implementation of the first scavenger prototype presented an air gap equal to $1.25 \mathrm{~mm}$ which is greater than the nominal value. This fact has a significant impact on the system performances as shown in Fig. 18. Comparing the data of the power generated by the wet test rig with those of the dry test rig, it is noticed a further drop of performance of the generated power. It goes from $9.1 \mathrm{~mW}$ produced at $1000 \mathrm{rpm}$ on the dry test rig to $5.2 \mathrm{~mW}$ 
generated by the wet test rig. This decay can be explained with stick-slip phenomena present among rotating and non rotating parts of the turbine, and it will be addressed and fixed in future studies.

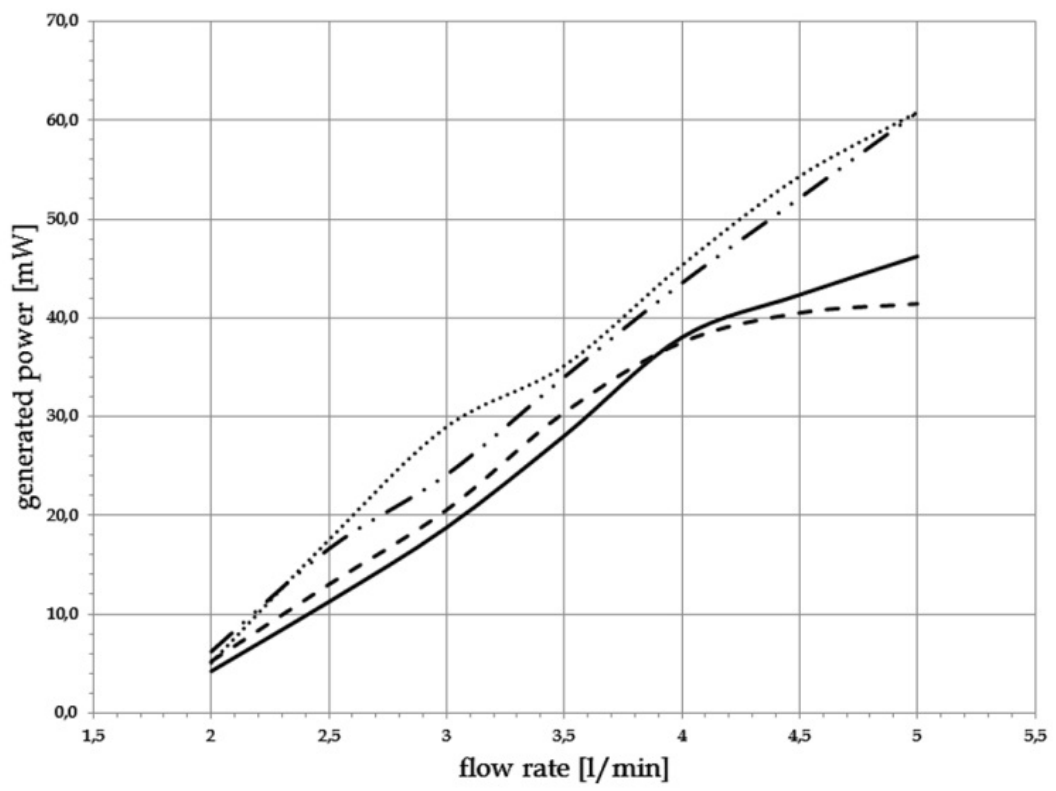

Figure 18. Wet Test Rig: generated power with external load equal to generator resistance. Nominal $N=1200$ (dashed line), $N=800$ (dash dotted line), $N=620$ (dotted line) and $N=490$ (solid line).

\section{Conclusions}

This study presents the trade-off analysis, the design, and the experimental validation of an Hydraulic Energy Scavenger applied to a motorized valve for domestic heating systems. The trade-off analysis conducted on the hydraulic and electric machines has identified the Banki turbine coupled to a claw poles generator as the solution to investigate and design. In this configuration the axis of rotation of the machine results to be perpendicular to the flow of the water thereby limiting problems of choking. The models underlying the design are validated from the electrical perspective by the dry test rig, and for the whole system by the wet test rig. The investigation was performed for different values of the air gap $t$, of the number of coils $\mathrm{N}$, and of the resistive load of the device. The comparison between model and experiments show a good correlation, even though the power generated by the device resulted to be lower than the desired design value. This lower power production is essentially related to manufacturing issues, namely an higher value of the air gap and a lower value of the permanent magnet induction. However, the good correlation between the experimental and theoretical data makes it possible to predict the achievement of the desired performance in case the indicated designed parameters are respected. 


\section{Author details}

Enrico Zenerino, Joaquim Girardello Detoni and Andrea Tonoli

Department of Mechanical and Aerospace Engineering, Politecnico di Torino, Italy

Mechatronics Lab, Politecnico di Torino, Italy

Marcello Chiaberge

Department of Electronics and Telecommunications, Politecnico di Torino, Italy

Mechatronics Lab, Politecnico di Torino, Italy

Diego Boero

Mechatronics Lab, Politecnico di Torino, Italy

\section{References}

Arnold, D.P.; (2007). Review of Microscale Magnetic Power Generation, IEEE Transactions on Magnetics, Vol. 43, No. 11, pp. (3940-3950).

Bansal, A.; Howey, D.A.; Holmes, A.S. (2009) - CM-Scale Air Turbine and Generator for Energy Harvesting from Low-Speed Flows, IEEE, ISBN 978-1-4244-4193-8

Chunyan, M.; Gengxin, L. (2010). Research on a self powered wireless ultrasonic flow sensor system, IEEE, ISBN 978-1-4244-6789-1

Hendershot, J. R.; Miller T. J. E. (1994). Design of brushless permanent-magnet motors, Magna Physics Publishing.

Inversin, A.(1994). Micro-Hydropowe Soucebook, A Pratical Guide to Design and Implementation in in Developing Cuntries, Third Printing

Kim, S.; Ji, C.; Galle, P.; Herrault, F.; Wu, X.; Lee, J.; Choi, C and Allen, M. G. (2010). An electromagnetic energy scavenger. Journal of Micromechanics and Microengineering, Vol.19, (August 2009), pp. (1-8), ISSN 0960-1317/09/094010

Lineykin, S.; Ben-Yaakov, S. (2007). Modeling and Analysis of Thermoelectric Modules, IEEE Transactions On Industry Application, Vol.43, No.2, pp. (505-512)

Lossec, M.; Multon, B and Ahmed, H.B. (2010). Micro-kinetic Generator: Modeling, Energy Conversion Optimization and Design Considerations Proceedings of the IEEE Mediterranean Electrotechnical Conference, pp. (1516-1521).

Lu, X.; Shuang-Hua, Y. (2010). Thermal energy harvesting for WSNs

Nechleba, M (1957). Hydraulic Turbines. Their Design and Equipment. ATIA ,Prague

Valdes, L.C. (2004). Competitive solar heat engines, Renewable energy, Vol. 29, pp. 1825-1842

Yan, T.C. ; Ibrahim, T. ; Nor, N. M. (2011). Micro Hydro Generator Applied on Domestic Pipeline, IEEE, ISBN 978-4577-0752-0

Zainuddin, H. ; Yahaya, M. S. ; Lazi, J. M. ; Basar, F. M. ; Ibrahim, Z. (2009). Design and Development of Pico-hydro Generator System for Energy Storage Using Consuming Water Distributed to Houses, World Academy of Science, Engineering and Technology, Vol.59, pp. (154-159) 